\title{
Normal values for nuclear cardiology: Japanese databases for myocardial perfusion, fatty acid and sympathetic imaging and left ventricular function
}

\author{
Kenichi Nakajima
}

Received: 15 December 2009/Accepted: 15 December 2009/Published online: 29 January 2010

(C) The Japanese Society of Nuclear Medicine 2010

\begin{abstract}
Myocardial normal databases for stress myocardial perfusion study have been created by the Japanese Society of Nuclear Medicine Working Group. The databases comprised gender-, camera rotation range- and radiopharmaceutical-specific data-sets from multiple institutions, and normal database files were created for installation in common nuclear cardiology software. Based on the electrocardiography-gated single-photon emission computed tomography (SPECT), left ventricular function, including ventricular volumes, systolic and diastolic functions and systolic wall thickening were also analyzed. Normal databases for fatty acid imaging using ${ }^{123}$ I-betamethyl-iodophenyl-pentadecanoic acid and sympathetic imaging using ${ }^{123}$ I-meta-iodobenzylguanidine were also examined. This review provides lists and overviews of normal values for myocardial SPECT and ventricular function in a Japanese population. The population-specific approach is a key factor for proper diagnostic and prognostic evaluation.
\end{abstract}

Keywords Normal database - Myocardial perfusion imaging - Systolic and diastolic function .

${ }^{123}$ I-BMIPP database $\cdot{ }^{123}$ I-MIBG database

In nuclear cardiology, myocardial perfusion single-photon emission computed tomography (SPECT) plays important roles for diagnosis, guiding therapeutic strategies,

\footnotetext{
K. Nakajima ( $\square)$

Department of Nuclear Medicine, Faculty of Medicine, Institute of Medical, Pharmaceutical and Health Sciences, Kanazawa University, 13-1 Takara-machi, Kanazawa 920-8641, Japan

e-mail: nakajima@med.kanazawa-u.ac.jp
}

evaluation of therapeutic effects and in predicting future cardiac events. Although recent technological advances in cardiac imaging modalities are remarkable, nuclear cardiology guidelines have been made in the United States and Japan, and a large amount of evidence has supported the use of radionuclide imaging in patients with acute syndromes, chronic syndromes and heart failure [1, 2]. Practical approaches for specific populations have also been recommended in nuclear cardiology guidelines.

The first step of image-based diagnosis is careful visual interpretation of myocardial images. Quantitative evaluation using myocardial perfusion imaging, however, aids diagnostic accuracy and enhances diagnostic certainty by reducing borderline interpretations. Various software programs have been developed, and more sophisticated and user-friendly approaches have been under development [3-5]. Common quantitative features of these software programs included calculating myocardial count on SPECT slices using appropriate segmentations and displaying the quantitative values on polar maps or threedimensional surface maps. In the processing algorithm, individual patient data were compared with control databases from low likelihood of heart diseases. Therefore, characteristics of the standard database might have influenced the final diagnostic accuracy. The Japanese Society of Nuclear Medicine (JSNM) database was created as a working group activity from these background studies [6].

To diagnose Japanese patients, it is necessary to use a normal Japanese database-this is a simple assumption. However, is it actually critical for the diagnosis? In this review, we deal with this question and summarize the characteristics of the Japanese databases for myocardial perfusion, fatty acid imaging and sympathetic imaging. In addition, characteristics of left ventricular function based 
on a gated SPECT study are reviewed in a Japanese population.

\section{Myocardial perfusion database}

Myocardial perfusion imaging with either exercise or pharmacological stress provides fundamental information for diagnosis of ischemic heart diseases, and its clinical utility has been established based on nearly three decades of experience. Although an individual institution-specific normal database may best fit the acquisition method and study population, accumulating and validating normal databases usually require cumbersome steps. The major merit of the authorized common databases, if they are available, is that they can be safely used in most of the institutions where standard acquisition and stress protocols are used. In the working group activity involving multiple nuclear medicine centers; however, we could not add all our raw data into one common database. Considering the factors that influenced regional myocardial distribution, we separated databases by genders, $360^{\circ}$ and $180^{\circ}$ rotation acquisitions, and ${ }^{201} \mathrm{Tl}$ and ${ }^{99} \mathrm{~m} \mathrm{Tc}$ pharmaceuticals. Because ${ }^{99 \mathrm{~m}}$ Tc-tetrofosmin and ${ }^{99 \mathrm{~m}} \mathrm{Tc}$-methoxy-isobutyl isonitrile (MIBI) showed no statistical differences in tracer distribution by preliminary investigation, both ${ }^{99 \mathrm{~m}} \mathrm{Tc}$ data types were added.

Nine hospitals collaborated on the normal database accumulation, and a total of 326 sets of exercise-rest myocardial perfusion images were accumulated from subjects with a low likelihood of cardiac diseases [6]. Using a DICOM (digital imaging and communications in medicine) format, projection images with gated and non-gated studies were anonymously transferred to the core laboratory of Kanazawa University. The reconstruction parameters for SPECT images were standardized and processed using the same computer (GE Yokogawa Medical Systems, Co, Ltd, Tokyo, Japan). The following software was available: QGS-QPS (Cedars-Sinai Medical Center, Los Angeles, CA, USA), Emory's Cardiac Toolbox (Emory University, Atlanta, GA, USA) and 4D-MSPECT (University of Michigan Medical Center, Ann Arbor, MI, USA). Although we have created standard database files for each software program, all the quantitative analyses written in this article are based on the QPS-QGS algorithm of quantification.

Figure 1 shows polar maps created by a normal Japanese population. When the $360^{\circ}$ and $180^{\circ}$ rotation methods were compared, septal segments showed higher values in the $180^{\circ}$ rotation for both ${ }^{99 \mathrm{~m}} \mathrm{Tc}$ and ${ }^{201} \mathrm{Tl}$. Differences in genders were significant in the mid- and apical inferior segments showing lower values in men due to diaphragmatic attenuation. In females, apical anterior segments showed lower values due to breast attenuation. Differences between ${ }^{201} \mathrm{Tl}$ and ${ }^{99 \mathrm{~m}} \mathrm{Tc}$ tracers were not negligible [6].

When the normal databases from the United States (Cedars-Sinai Medical Center database pre-installed in the standard QPS) and Japan were compared, attenuation in the anterior wall was greater in the American female population than in the Japanese population. Inferior diaphragmatic attenuation was greater in the American population in males. Does the small difference between nations actually influence the diagnostic accuracy? The diagnostic performance was evaluated by receiver-operating characteristic analysis among Japanese $360^{\circ}$ and $180^{\circ}$ acquisition databases (Jp360 and Jp180) and an American database with a $180^{\circ}$ acquisition database (US180), when Japanese patients with a $360^{\circ}$ acquisition method were analyzed [7]. Areas under the curve were the highest for Jp360 (0.842), followed by Jp180 (0.758) and US180 (0.728) databases $(P=0.019, \quad J p 360$ vs. US180; $P=0.035$, Jp360 vs. Jp180). Because of the database difference in the inferior wall of male patients, diagnostic improvement was demonstrated in the right coronary artery territory (Fig. 2). Similarly, differences in the anterior wall in female patients resulted in improved accuracy in left anterior descending coronary territory. Thus, it was essential to use populationand acquisition-specific databases when using quantitative perfusion SPECT software. Clinical validation was subsequently performed when the same database could be used in various types of software and versions [8]. Five institutions participated in the study, and each used different acquisition settings that included $360^{\circ}$ and $180^{\circ}$ rotation ranges, camera configurations and camera orbits. The core laboratory created normal database files from the original JSNM short-axis image sets, which were fitted for each software version. Overall sensitivity, specificity and accuracy of patient-based analysis were 77, 72 and $75 \%$, respectively, based on the quantitative analysis using the common database, whereas those by institutional visual expert reading were 72,79 and $75 \%$, respectively. Although the diagnostic accuracy was moderately good, the study indicated that the common database could be applicable to different institutions when such a database was appropriately created according to the softwarespecific database generator.

\section{Left ventricular function in a Japanese population}

A number of studies have demonstrated that gated SPECT evaluation of ejection fraction (EF), regional wall motion and wall thickening was accurate, and gated SPECT could be considered as an effective substitute modality of radionuclide ventriculography for the assessment of left ventricular function [9-12]. Although the reproducibility of 


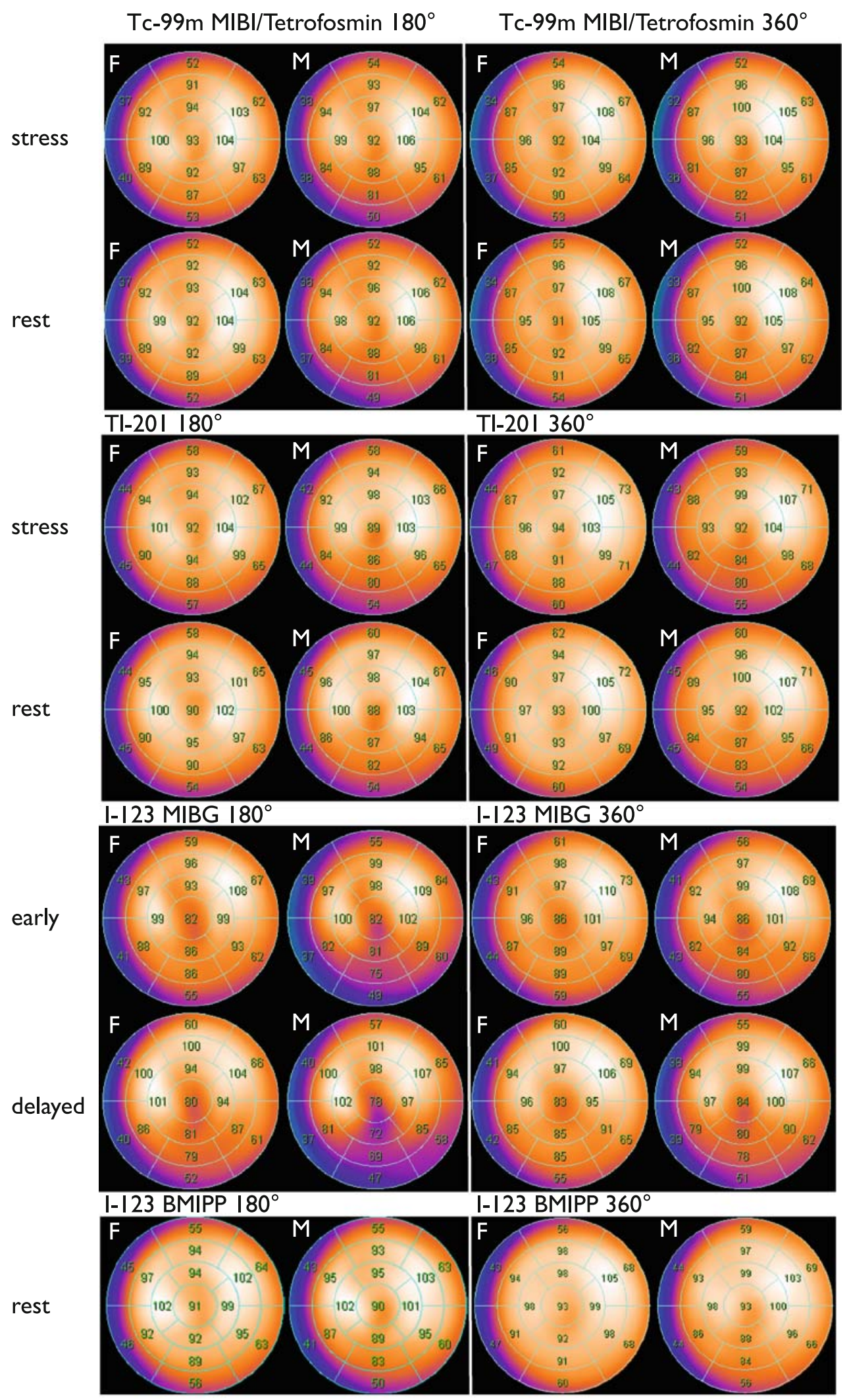

Fig. 1 Polar map presentation of normal myocardial SPECT databases using a 17-segment model. Average segmental values are overlaid on the maps. Upper two panels stress and rest perfusion studies with ${ }^{99 \mathrm{~m}} \mathrm{Tc}$-labeled tracers (MIBI and tetrofosmin) and ${ }^{201} \mathrm{Tl}$ using $180^{\circ}$ and $360^{\circ}$ acquisition methods. Lower two panels ${ }^{123} \mathrm{I}$ BMIPP and ${ }^{123} \mathrm{I}$ MIBG studies with $180^{\circ}$ and $360^{\circ}$ acquisition methods 
A Scores of RCA region in male patients

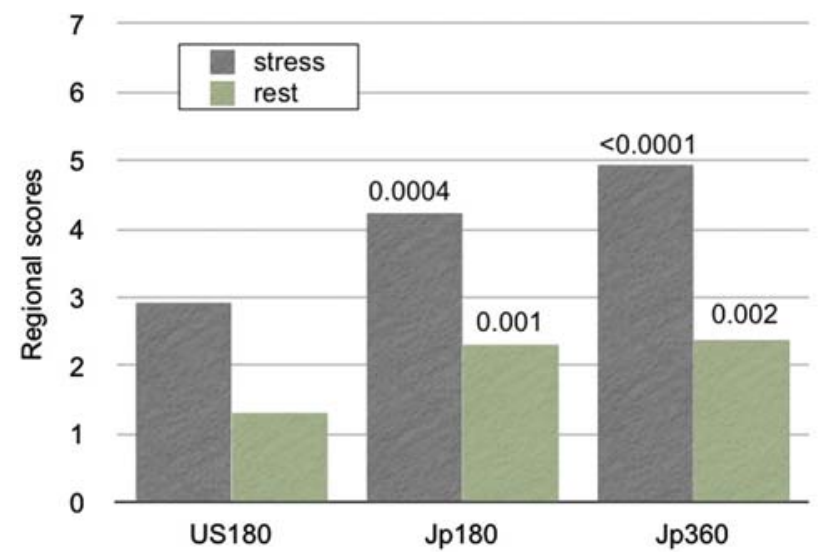

B Scores of $L A D$ region in female patients

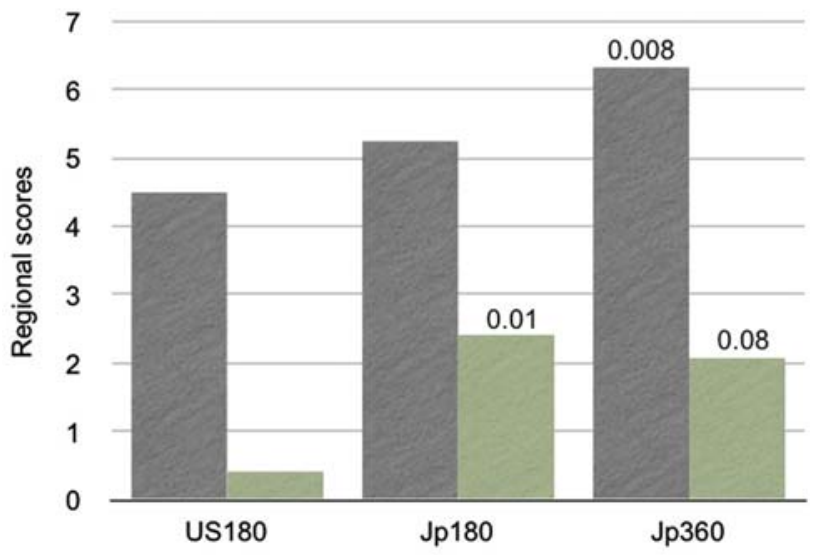

Fig. 2 Regional summed stress scores determined by Japanese $180^{\circ}$ and $360^{\circ}$ databases (Jp180, Jp360) and an American $180^{\circ}$ database (US180). The normal database was applied to a Japanese patient group $(n=90)$ with a $360^{\circ}$ acquisition [7]. a Regional scores in the right coronary artery (RCA) territory in males. b Those in the left anterior descending coronary artery (LAD) territory in females. A $P$ value in each bar is indicated in comparison with US180 database

QGS software was evaluated well regarding inter-observer variability, the precision of $\mathrm{EF}$ and left ventricular volume determination was further confirmed in the multicenter J-ACCESS investigation [13, 14]. The reproducibility or precision of EF was good in 106 hospitals, showing a standard deviation of $<3.6 \%$, and the coefficient of variance of the end-diastolic volume was $<9.3 \%$ in all cases. However, it has also been noticed that if American and European standard values are used, the normal values were not always appropriate for a Japanese population in clinical practice [15-18]. In fact, when a certain threshold value was used for discriminating abnormal patients or estimating cardiac event risks, it may have been potentially misleading for patient care. The normal values of left ventricular function calculated in a Japanese population are shown in Table 1, which are derived from the JSNM
Table 1 Normal values of left ventricular function by gated SPECT based on 16-frame gated SPECT with ${ }^{99 \mathrm{~m}} \mathrm{Tc}-\mathrm{MIBI}$ and tetrofosmin

\begin{tabular}{|c|c|c|}
\hline JSNM WG database & Males & Females \\
\hline Number of subjects & 49 & 35 \\
\hline \multicolumn{3}{|l|}{ Age (years) } \\
\hline Mean $\pm \mathrm{SD}$ & $58.3 \pm 13.0$ & $55.6 \pm 13.7$ \\
\hline \multicolumn{3}{|l|}{$\mathrm{EF}(\%)$} \\
\hline Mean \pm SD & $65.6 \pm 5.9$ & $71.0 \pm 7.3$ \\
\hline Mean - 2SD & 53.8 & 56.4 \\
\hline Mean $+2 \mathrm{SD}$ & 77.4 & 85.6 \\
\hline \multicolumn{3}{|l|}{ EDV (mL) } \\
\hline Mean $\pm \mathrm{SD}$ & $82.3 \pm 16.3$ & $67.4 \pm 13.1$ \\
\hline Mean - 2SD & 49.7 & 41.2 \\
\hline Mean $+2 \mathrm{SD}$ & 114.9 & 93.6 \\
\hline \multicolumn{3}{|l|}{ ESV (mL) } \\
\hline Mean $\pm \mathrm{SD}$ & $28.7 \pm 9.0$ & $20.1 \pm 7.9$ \\
\hline Mean - 2SD & 10.7 & 4.3 \\
\hline Mean +2 SD & 46.7 & 35.9 \\
\hline \multicolumn{3}{|l|}{ EDVI $\left(\mathrm{mL} / \mathrm{m}^{2}\right)$} \\
\hline Mean \pm SD & $49.0 \pm 8.0$ & $42.3 \pm 5.9$ \\
\hline Mean - 2SD & 33.0 & 30.5 \\
\hline Mean +2 SD & 65.0 & 54.1 \\
\hline \multicolumn{3}{|l|}{$\operatorname{ESVI}\left(\mathrm{mL} / \mathrm{m}^{2}\right)$} \\
\hline Mean \pm SD & $17.1 \pm 4.8$ & $12.6 \pm 4.0$ \\
\hline Mean - 2SD & 7.5 & 4.6 \\
\hline Mean + 2SD & 26.7 & 20.6 \\
\hline Small heart $(\mathrm{ESV}<20 \mathrm{~mL})$ & $22 \%$ & $51 \%$ \\
\hline
\end{tabular}

JSNM WG Japanese Society of Nuclear Medicine Working Group, EF ejection fraction, $E D V$ end-diastolic volume, $E S V$ end-systolic volume, EDVI EDV index, ESVI ESV index

working group study. The calculated EF value was higher for women than in men in Japanese populations for both JSNM database and J-ACCESS prognostic database (Table 2) [6, 19]. This is not simply because of underestimation of volume and overestimation of $\mathrm{EF}$ in small hearts due to an algorithm of edge tracing by QGS software $[20,21]$. A magnetic resonance imaging (MRI) study also demonstrated that women had a higher left ventricular EF than men in the general population of Dallas County residents aged 30-65 years (1435 women, 1183 men) [22]. A low left ventricular EF was defined as below $61 \%$ in women and below 55\% in men by MRI, and the difference between genders was comparable for our gated SPECT studies. In a Japanese population, mean female EF was higher when compared with that of American and European populations, which seemed to reflect smaller body size in Japanese women (Table 2). The lower borders of EF can be defined as below $\sim 55 \%$ in women and below $\sim 50 \%$ in men using gated SPECT.

The diastolic function determined for the Japanese population using a 16-frame format is summarized in 
Table 2 Comparison of normal values of left ventricular function by QGS software

\begin{tabular}{|c|c|c|c|c|c|c|}
\hline & \multicolumn{3}{|l|}{ Males } & \multicolumn{3}{|l|}{ Females } \\
\hline & Mean \pm SD & Mean - 2SD & Mean +2 SD & Mean \pm SD & Mean - 2SD & Mean +2 SD \\
\hline \multicolumn{7}{|c|}{ De Bondt (43 M, 59 F) [16] } \\
\hline $\mathrm{EF}(\%)$ & $59 \pm 6$ & 47 & 71 & $66 \pm 9$ & 48 & 84 \\
\hline EDV (mL) & $106 \pm 25$ & 56 & 156 & $75 \pm 23$ & 29 & 121 \\
\hline ESV (mL) & $44 \pm 14$ & 16 & 72 & $27 \pm 14$ & -1 & 55 \\
\hline $\operatorname{EDVI}\left(\mathrm{mL} / \mathrm{m}^{2}\right)$ & $54 \pm 14$ & 26 & 82 & $43 \pm 11$ & 21 & 65 \\
\hline $\operatorname{ESVI}\left(\mathrm{mL} / \mathrm{m}^{2}\right)$ & $23 \pm 8$ & 7 & 39 & $16 \pm 7$ & 2 & 30 \\
\hline \multicolumn{7}{|c|}{ Ababneh (116 M, 124 F) [17] } \\
\hline $\mathrm{EF}(\%)$ & $62 \pm 7$ & 48 & 76 & $67 \pm 8$ & 51 & 83 \\
\hline EDV (mL) & $74 \pm 22$ & 30 & 118 & $57 \pm 17$ & 23 & 91 \\
\hline ESV (mL) & $29 \pm 13$ & 3 & 55 & $19 \pm 11$ & -3 & 41 \\
\hline $\operatorname{EDVI}\left(\mathrm{mL} / \mathrm{m}^{2}\right)$ & $38 \pm 9$ & 20 & 56 & $32 \pm 8$ & 16 & 48 \\
\hline $\operatorname{ESVI}\left(\mathrm{mL} / \mathrm{m}^{2}\right)$ & $15 \pm 6$ & 3 & 27 & $10 \pm 5$ & 0 & 20 \\
\hline \multicolumn{7}{|c|}{ J-ACCESS (119 M, 149 F) [14] } \\
\hline $\mathrm{EF}(\%)$ & $63 \pm 7$ & 49 & 77 & $74 \pm 9$ & 56 & 92 \\
\hline EDV (mL) & $88 \pm 23$ & 44 & 132 & $59 \pm 17$ & 25 & 93 \\
\hline $\mathrm{ESV}(\mathrm{mL})$ & $33 \pm 13$ & 7 & 59 & $17 \pm 10$ & -3 & 37 \\
\hline $\operatorname{EDVI}\left(\mathrm{mL} / \mathrm{m}^{2}\right)$ & $51 \pm 12$ & 27 & 75 & $39 \pm 11$ & 17 & 61 \\
\hline ESVI $\left(\mathrm{mL} / \mathrm{m}^{2}\right)$ & $19 \pm 7$ & 5 & 33 & $11 \pm 6$ & -1 & 23 \\
\hline \multicolumn{7}{|c|}{ JSNM WG (68 M and $68 \mathrm{~F})[6]^{\mathrm{a}}$} \\
\hline $\mathrm{EF}(\%)$ & $64 \pm 7$ & 50 & 78 & $69 \pm 7$ & 54 & 84 \\
\hline EDV (mL) & $80 \pm 16$ & 49 & 112 & $64 \pm 13$ & 39 & 90 \\
\hline ESV (mL) & $29 \pm 9$ & 12 & 47 & $20 \pm 7$ & 7 & 34 \\
\hline $\operatorname{EDVI}\left(\mathrm{mL} / \mathrm{m}^{2}\right)$ & $47 \pm 9$ & 30 & 64 & $42 \pm 7$ & 29 & 55 \\
\hline ESVI $\left(\mathrm{mL} / \mathrm{m}^{2}\right)$ & $17 \pm 5$ & 8 & 27 & $13 \pm 4$ & 5 & 22 \\
\hline
\end{tabular}

$M$ males, $F$ females

${ }^{\text {a }}$ Both data from $180^{\circ}$ and $360^{\circ}$ rotation acquisition methods are added

Table 3 using the JSNM database with two previously published studies [18, 23]. Although a 32-frame division of a cardiac cycle might provide better correlation with those determined by radionuclide ventriculography [24, 25], a more practical approach seems to be a 16-frame acquisition per RR interval, when considering the counting statistics of the myocardial activity. Using QGS software algorithm, abnormal thresholds in the American population included peak filling rate (PFR) $<1.70$ per $\mathrm{s}$ and time to PFR (TTPF) $>208 \mathrm{~ms}$ [18]. Multivariable analysis showed that age, sex, EF and heart rate were strong predictors for PFR, whereas TTPF was not influenced by any clinical or systolic function variables. The Japanese population also showed comparable normal values (Table 3 ). When patients were classified into two age groups of $<60$ and $\geq 60$ years, standard deviations of PFR, $1 / 3$ mean filling rate, TTPF and TTPF/RR were larger in the older group than in the younger group. Because age-related differences were observed, it should be kept in mind that diastolic dysfunction in elderly patients and in those with $\mathrm{EF}<50 \%$ are more common [26]. Large population-based criteria, adjusted for age and sex, for normal versus abnormal diastolic function have not yet been established. Therefore, when the severity of diastolic dysfunction has become increasingly important in patients with the clinical syndrome of heart failure, the validity of diastolic function should be more extensively evaluated with nuclear methods [1].

To evaluate left ventricular regional wall motion, wall thickening seemed to be more appropriate for gated SPECT. When wall motion defined by endocardial perimeter was used (unit of $\mathrm{mm}$ ), septal motion was relatively low when compared with that of free walls. The postoperative heart typically showed decreased motion even when septal systolic wall thickening (unit of \%) was preserved [27-29]. Therefore, we recommend the use of thickening map for evaluating regional asynergy. Normal values of wall thickening were evaluated from a total of 202 patients in the JSNM database [30]. In any acquisition protocol, myocardial wall thickening in the apex was higher than that in the mid and basal regions. Different rotation angles showed no significant change on wall 
Table 3 Normal values of diastolic parameters using 16-frame gated SPECT

\begin{tabular}{|c|c|c|c|c|c|}
\hline & \multicolumn{3}{|l|}{ JSNM WG } & \multirow{2}{*}{$\begin{array}{l}\text { Nakajima [23] } \\
\text { Control group }\end{array}$} & \multirow{2}{*}{$\begin{array}{l}\text { Akincioglu [18] } \\
\text { All }\end{array}$} \\
\hline & All & $<60$ years & $\geq 60$ years & & \\
\hline Number of subjects & 60 & 33 & 27 & 16 & 90 \\
\hline Age $($ mean \pm SD) & $58 \pm 15$ & $48 \pm 11$ & $70 \pm 6$ & $50 \pm 12$ & $53 \pm 11$ \\
\hline Heart rate (per min) $($ mean $\pm \mathrm{SD})$ & $66 \pm 12$ & $66 \pm 11$ & $65 \pm 13$ & $65 \pm 7$ & $74 \pm 8$ \\
\hline \multicolumn{6}{|l|}{ Peak filling rate (per s) } \\
\hline Mean \pm SD & $2.69 \pm 0.57$ & $2.79 \pm 0.53$ & $2.58 \pm 0.60$ & $2.46 \pm 0.45$ & $2.62 \pm 0.46$ \\
\hline Mean - 2SD & 1.55 & 1.73 & 1.38 & 1.56 & 1.70 \\
\hline Mean + 2SD & 3.83 & 3.85 & 3.78 & 3.36 & 3.54 \\
\hline \multicolumn{6}{|l|}{$1 / 3$ mean filling rate (per $s$ ) } \\
\hline Mean \pm SD & $1.60 \pm 0.39$ & $1.68 \pm 0.30$ & $1.51 \pm 0.47$ & $1.52 \pm 0.25$ & \\
\hline Mean - 2SD & 0.82 & 1.08 & 0.57 & 1.02 & \\
\hline Mean + 2SD & 2.38 & 2.28 & 2.45 & 2.02 & \\
\hline \multicolumn{6}{|l|}{ Time to peak filling $(\mathrm{ms})^{\mathrm{a}}$} \\
\hline Mean \pm SD & $167 \pm 38$ & $159 \pm 26$ & $176 \pm 48$ & $166 \pm 22$ & $165 \pm 22$ \\
\hline Mean - 2SD & 91 & 108 & 80 & 122 & 121 \\
\hline Mean + 2SD & 243 & 210 & 273 & 210 & 208 \\
\hline \multicolumn{6}{|l|}{ Time to peak filling/RR interval } \\
\hline Mean \pm SD & $0.18 \pm 0.03$ & $0.17 \pm 0.02$ & $0.18 \pm 0.04$ & $0.18 \pm 0.02$ & \\
\hline Mean - 2SD & 0.12 & 0.13 & 0.11 & 0.14 & \\
\hline Mean +2 SD & 0.24 & 0.22 & 0.26 & 0.22 & \\
\hline \multicolumn{6}{|l|}{ Ejection fraction $(\%)$} \\
\hline Mean \pm SD & $68 \pm 6$ & $67 \pm 7$ & $69 \pm 7$ & $68 \pm 9$ & $64 \pm 6$ \\
\hline
\end{tabular}

${ }^{a}$ Time to peak filling is defined as time from end systole to PFR

thickening, but different frame rates and tracers showed significant differences in both genders. The higher wall thickening in women than in men was comparable to findings of the higher EF in women. Because wall thickening has not been used separately depending on the acquisition protocol and gender differences so far, appropriate database construction might be required. Because wall thickening was relatively uniform in the six basal segments, six mid segments and four apical segments in each gender, practical threshold values are shown in Table 4 using 16-frame gated SPECT with both $360^{\circ}$ and $180^{\circ}$ acquisition methods added. For males, approximate lower borders of wall thickening are 30, 40 and 50\% for mid, apical and apex segments, respectively. For females, they were 35,45 and $55 \%$ for mid, apical and apex segments, respectively.

\section{Normal database for I-123 BMIPP fatty acid imaging}

In clinical practices in Japan, where BMIPP is approved by the Health, Labor and Welfare Ministry and covered by health insurance, normal myocardial ${ }^{123} \mathrm{I}$-BMIPP databases
Table 4 Normal values of wall thickening (\%) using 16-frame gated SPECT

\begin{tabular}{lll}
\hline & Males & Females \\
\hline $\begin{array}{l}\text { Number of subjects } \\
\text { Six basal segments }\end{array}$ & 36 & 33 \\
Mean \pm SD & $24.7 \pm 9.5$ & $25.1 \pm 9.4$ \\
Mean - 2SD & 5.7 & 6.2 \\
Mean + 2SD & 43.8 & 43.9 \\
Six mid segments & & \\
Mean \pm SD & $47.2 \pm 9.2$ & $53.0 \pm 9.1$ \\
Mean - 2SD & 28.8 & 34.8 \\
Mean + 2SD & 65.5 & 71.3 \\
Four apical segments & & \\
Mean \pm SD & $62.6 \pm 10.5$ & $69.1 \pm 11.3$ \\
Mean -2 SD & 41.7 & 46.5 \\
Mean + 2SD & 83.5 & 91.7 \\
An apex segment & & \\
Mean \pm SD & $74.2 \pm 12.1$ & $77.5 \pm 12.1$ \\
Mean $-2 S D$ & 50.0 & 53.4 \\
Mean $+2 S D$ & 98.4 & 101.7 \\
\hline
\end{tabular}

Both data from $180^{\circ}$ and $360^{\circ}$ rotation acquisition methods are added [30] 
have not been defined and utilized. Japanese nuclear cardiology guidelines approved fatty acid imaging with respect to indications for acute coronary syndromes and repeated ischemic episodes in chronic syndromes [2, 31-33]. Common findings included perfusion and metabolic mismatch, suggesting temporary metabolic deterioration in the viable myocardium. The BMIPP accumulation reflected a risk area after acute myocardial infarction and perfusion defect reflected myocardial infarction [34]. Although comparison between perfusion and metabolism is essential, an appropriate normal database would contribute to enhanced diagnostic accuracy. Owing to small, but significant differences between ${ }^{99 \mathrm{~m}} \mathrm{Tc}$ perfusion tracer and ${ }^{123}$ I-BMIPP existed, the use of common databases would be helpful for accurate diagnosis [35]. In the ${ }^{123}$ I-labeled tracer databases, only $360^{\circ}$ datasets were available. As a result, $180^{\circ}$ databases were made tentatively using a left anterior $180^{\circ}$ arc of the whole rotation range (Fig. 1).

\section{Normal database for I-123 MIBG sympathetic imaging}

According to nuclear cardiology guidelines by the Japanese Circulation Society, ${ }^{123}$ I-MIBG contains clinical indications for heart failure particularly for evaluating severity, therapeutic effects and prognosis of heart failure [2, 36-38]. Using MIBG uptake indices, a quantitative threshold for low risk of cardiac mortality and potentially fatal ventricular arrhythmias might be identified [39]. In addition, the MIBG study showed markedly decreased myocardial uptake in Parkinson's disease, dementia with Lewy bodies (DLB) and pure autonomic failure, which is a common feature of Lewybody diseases [40-43]. In the report of the Consortium on DLB International Workshop, the MIBG study was included as one of the supportive features of DLB [44]. To quantify MIBG uptake, heart to mediastinal average count (H/M) ratio has been used as a simple method that could be used in any institutes [45]. However, lack of standardization hampers large-scale implementation of ${ }^{123}$ I-MIBG parameters in the evaluation of patients with chronic heart failure [46, 47]. In particular, a choice of collimators was an important factor of quantitative variations. Thus, the JSNM database also aimed at creating a normal database for planar anterior images as well as SPECT imaging.

A number of MIBG studies have used the $\mathrm{H} / \mathrm{M}$ ratio as a practical method for quantification. When low-energy collimators were used, the average $\mathrm{H} / \mathrm{M}$ ratios were around 2.1-2.4, with the lower cutoff values around 1.6-2.0 [48]. In contrast, if medium-energy or low-medium-energy collimators were used, the average values were around 2.8-3.0. The H/M ratio accumulated from the JSNM working group is summarized in Table 5. According to a
Table 5 Normal values of I-123 MIBG heart to mediastinum ratio

\begin{tabular}{lll}
\hline & LE collimator & $\begin{array}{l}\text { ME or LME } \\
\text { collimator }\end{array}$ \\
\hline JSNM WG data & \\
Number of patients & 36 & 20 \\
Early H/M ratio & & \\
Mean \pm SD & $2.39 \pm 0.21$ & $2.76 \pm 0.31$ \\
Mean - 2SD & 1.97 & 2.14 \\
Mean + 2SD & 2.81 & 3.38 \\
Delayed H/M ratio & & \\
Mean \pm SD & $2.49 \pm 0.25$ & $3.01 \pm 0.35$ \\
Mean -2 SD & 1.99 & 2.31 \\
Mean + 2SD & 2.99 & 3.71 \\
Questionnaire survey in Japan $[47]$ & \\
Number of institution & 15 & 2 \\
Early H/M ratio & & \\
Mean \pm SD & $2.25 \pm 0.27$ & $2.78 \pm 0.32$ \\
Mean -2 SD & 1.71 & 2.14 \\
Mean $+2 S D$ & 2.79 & 3.42 \\
Delayed H/M ratio & & $3.17 \pm 0.29$ \\
Mean \pm SD & $2.36 \pm 0.24$ & \\
Mean -2 SD & 1.88 & 2.59 \\
Mean $+2 S D$ & 2.84 & \\
\hline
\end{tabular}

$L E$ low energy, $M E$ medium energy, $L M E$ low-medium-energy, $H / M$ heart to mediastinum

questionnaire survey conducted in Japan, normal H/M ratios using a low-energy high-resolution collimator and a medium-energy collimator were comparable to those of the JSNM database [49]. Significant differences were found between $\mathrm{H} / \mathrm{M}$ values from data using both collimators. To standardize H/M ratios, some attempts have been presented, such as use of medium-energy collimators [50], deconvolution of septal penetration [51] and multiplewindow or iodine-123 dual energy window (IDW) methods $[48,52]$. When the IDW method was used with a calibration phantom and in clinical studies, corrected $\mathrm{H} / \mathrm{M}$ values with a low-energy collimator became comparable to that of a medium-energy collimator. A method for stabilizing the location of the region of interest should also be investigated. Further studies are required to determine whether this type of correction can truly enhance diagnostic ability, and whether they may be used for multicenter studies.

Three-dimensional MIBG distribution seemed to be heterogeneous based on a SPECT study [53]. The sympathetic nervous system in the human left ventricle showed age- and gender-related regional changes [54]. In clinical practice, a decrease in MIBG uptake in the inferolateral walls has sometimes been observed particularly in elderly patients, but individual differences in segmental uptake have also been observed. In accordance with this finding, 
myocardial MIBG databases showed relatively decreased activity in the inferior, which was more prominent in the delayed image (Fig. 1). The deviation of the apical inferior wall is relatively increased. Although it is conceivable that age-related changes influenced count distribution, we could not make age-specific standards because of the limited number of normal patients.

\section{Cardiovascular event risk}

Quantification of abnormal perfusion defect or induced ischemia serves to enhance diagnostic accuracy and patient risk stratification. For estimating patient prognosis, semiquantitative evaluation of summed score of stress, rest and difference are powerful predictors of hard and all events, in addition to being factors of baseline left ventricular function. The evidence-based role of scoring has been employed for risk evaluation in patients suspected of having ischemic heart disease. The Japanese multicenter prognostic cohort study (J-ACCESS prognostic database) demonstrated that a stress myocardial perfusion study with gated SPECT could provide effective prognostic information, although the hard event rate was relatively lower in a Japanese population [5557]. In fact, according to the governmental statistics, the mortality rate caused by cardiac diseases in Japan is one of the lowest in the world compared with that of American and European countries. The rate is about one-third that of the US. Nevertheless, normal and severely abnormal summed stress score values were associated with low (2.3\%/3 years) and high (9.2\%/3 years) rates of major cardiac events, including cardiac death, non-fatal myocardial infarction and severe heart failure. Rates of major cardiac events were significantly higher in patients with $\mathrm{EF}<45 \%$ than in those with $\mathrm{EF} \geq 45 \%$ (16.6 vs. $2.9 \% / 3$ years; $P<0.001$ ). Although a precise description for the event risk is not the aim of this article, risk evaluation based on the Japanese databases and ethnic background would be essential for effective patient management.

\section{Future directions}

The characteristics of normal databases depend on technological advances in SPECT. Several attempts have been performed to improve attenuation, scatter and resolution corrections [58, 59]. Attenuation correction might enable the use of a single gender-independent normal database and a single criterion for abnormality. However, attenuation correction seems to provide different effects based on the imaging system used [60]. The prone positioning was also used to compensate for attenuation artifacts [61, 62]. Attenuation correction of myocardial SPECT by scatter-photopeak window method was also investigated [63]. Because myocardial count distribution in normal subjects does not show complete homogeneity with any methods, some kind of additional database matched with advanced technological capability would be required. Moreover, a validation study to determine appropriate cutoff values between normal and abnormal groups should be investigated. With the recent advances of fusion technology using X-ray computed tomography angiography and myocardial perfusion imaging, functional relevance to coronary stenosis could be enhanced [64-66]. Quantitative accuracy would be further improved by anatomical and functional correlations using appropriate normal databases.

\section{Conclusions}

The JSNM normal databases were the first databases authorized by the scientific society. The mixture of typical acquisition condition from different institutions has merits for applying the same database to a multicenter study and to inter-institution comparison of the SPECT results. Although an institution-specific database created in every institution would be theoretically desirable, a common database could be a practical approach for clinical evaluation of patients suspected of having coronary artery diseases. Finally, normal values derived from a common database could be applied in clinical studies with respect to myocardial perfusion, left ventricular systolic and diastolic function, fatty acid imaging and sympathetic imaging.

\section{Appendix}

Participating hospitals and researchers for database accumulation

Chief of the working group: Kenichi Nakajima (Kanazawa University), Koichi Okuda, Shinro Matsuo*, Tatsuya Yoneyama, Junichi Taki* (Kanazawa University Hospital), Shinichiro Kumita*, Yoshimitsu Fukushima (Nippon Medical School Hospital), Yoshio Ishida* (National Cardiovascular Center), Jun Hashimoto* (Keio University Hospital, Tokai University at present), Mitsuru Momose* (Tokyo Women's Medical University), Koichi Morita*, Masayuki Inubushi, Keiichiro Yoshinaga* (Hokkaido University Hospital), Shohei Yamashina* (Toho University Omori Medical Center), Hirotaka Maruno* (Toranomon Hospital), Naoya Matsumoto* (Nihon University School of Medicine), Masaya Kawano* (Kanazawa Cardiovascular Center), Kazuyuki Sakata (Shizuoka Cancer Center), *JSNM working group member. 
Cooperation for creating normal databases

Guido Germano, Piotr Slomka, Geoff Pollard (CedarsSinai Medical Center, USA), Ernest Garcia (Emory University Hospital, USA), Edward Ficaro (University of Michigan Medical Center, USA).

\section{Cooperating companies}

GE Yokogawa Medical Systems (Tokyo, Japan), Toshiba Medical Systems Corporation (Tokyo, Japan), SiemensAsahi Medical Technologies (Tokyo, Japan), Hitachi Medical Corporation (Tokyo, Japan), Shimadzu Corporation (Kyoto, Japan), FUJIFILM RI Pharma (Tokyo, Japan), Nihon Medi-Physics (Tokyo, Japan).

\section{References}

1. Klocke FJ, Baird MG, Lorell BH, Bateman TM, Messer JV, Berman DS, et al. ACC/AHA/ASNC guidelines for the clinical use of cardiac radionuclide imaging-executive summary: a report of the American College of Cardiology/American Heart Association Task Force on Practice Guidelines (ACC/AHA/ASNC Committee to Revise the 1995 Guidelines for the Clinical Use of Cardiac Radionuclide Imaging). Circulation. 2003;108:1404-18.

2. Tamaki N. Guidelines for clinical use of cardiac nuclear medicine (JCS 2005). Circ J. 2005;69(Suppl. IV):1125-202.

3. Germano G, Kavanagh PB, Slomka PJ, Van Kriekinge SD, Pollard G, Berman DS. Quantitation in gated perfusion SPECT imaging: the Cedars-Sinai approach. J Nucl Cardiol. 2007; 14:433-54.

4. Garcia EV, Faber TL, Cooke CD, Folks RD, Chen J, Santana C. The increasing role of quantification in clinical nuclear cardiology: the Emory approach. J Nucl Cardiol. 2007;14:420-32.

5. Ficaro EP, Lee BC, Kritzman JN, Corbett JR. Corridor4DM: the Michigan method for quantitative nuclear cardiology. J Nucl Cardiol. 2007;14:455-65.

6. Nakajima K, Kumita S, Ishida Y, Momose M, Hashimoto J, Morita K, et al. Creation and characterization of Japanese standards for myocardial perfusion SPECT: database from the Japanese Society of Nuclear Medicine Working Group. Ann Nucl Med. 2007;21:505-11.

7. Nakajima K, Okuda K, Kawano M, Matsuo S, Slomka P, Germano $G$, et al. The importance of population-specific normal database for quantification of myocardial ischemia: comparison between Japanese 360 and 180-degree databases and a US database. J Nucl Cardiol. 2009;16:422-30.

8. Nakajima K, Matsuo S, Kawano M, Matsumoto N, Hashimoto J, Yoshinaga $\mathrm{K}$, et al. The validity of multi-center common normal database for identifying myocardial ischemia: Japanese Society of Nuclear Medicine working group database. Ann Nucl Med 2010 (in press)

9. Sciagra R. The expanding role of left ventricular functional assessment using gated myocardial perfusion SPECT: the supporting actor is stealing the scene. Eur J Nucl Med Mol Imaging. 2007;34:1107-22.

10. Kondo C, Fukushima K, Kusakabe K. Measurement of left ventricular volumes, ejection fraction by quantitative gated SPET, contrast ventriculography and magnetic resonance imaging: a meta-analysis. Eur J Nucl Med Mol Imaging. 2003;30:851-8.
11. Go V, Bhatt MR, Hendel RC. The diagnostic and prognostic value of ECG-gated SPECT myocardial perfusion imaging. J Nucl Med. 2004;45:912-21.

12. Nakajima K, Higuchi T, Taki J, Kawano M, Tonami N. Accuracy of ventricular volume and ejection fraction measured by gated myocardial SPECT: comparison of 4 software programs. J Nucl Med. 2001;42:1571-8.

13. Germano G, Kiat H, Kavanagh PB, Moriel M, Mazzanti M, Su HT, et al. Automatic quantification of ejection fraction from gated myocardial perfusion SPECT. J Nucl Med. 1995;36:2138-47.

14. Nakajima K, Nishimura T. Inter-institution preference-based variability of ejection fraction and volumes using quantitative gated SPECT with ${ }^{99 \mathrm{~m}}$ Tc-tetrofosmin: a multicentre study involving 106 hospitals. Eur J Nucl Med Mol Imaging. 2006;33:127-33.

15. Rozanski A, Nichols K, Yao SS, Malholtra S, Cohen R, DePuey EG. Development and application of normal limits for left ventricular ejection fraction and volume measurements from ${ }^{99 \mathrm{~m}} \mathrm{Tc}-$ sestamibi myocardial perfusion gates SPECT. J Nucl Med. 2000;41:1445-50.

16. De Bondt P, Van de Wiele C, De Sutter J, De Winter F, De Backer G, Dierckx RA. Age- and gender-specific differences in left ventricular cardiac function and volumes determined by gated SPET. Eur J Nucl Med. 2001;28:620-4.

17. Ababneh AA, Sciacca RR, Kim B, Bergmann SR. Normal limits for left ventricular ejection fraction and volumes estimated with gated myocardial perfusion imaging in patients with normal exercise test results: influence of tracer, gender, and acquisition camera. J Nucl Cardiol. 2000;7:661-8.

18. Akincioglu C, Berman DS, Nishina H, Kavanagh PB, Slomka PJ, Abidov A, et al. Assessment of diastolic function using 16-frame ${ }^{99 \mathrm{~m}}$ Tc-sestamibi gated myocardial perfusion SPECT: normal values. J Nucl Med. 2005;46:1102-8.

19. Nakajima K, Kusuoka H, Nishimura S, Yamashina A, Nishimura T. Normal limits of ejection fraction and volumes determined by gated SPECT in clinically normal patients without cardiac events: a study based on the J-ACCESS database. Eur J Nucl Med Mol Imaging. 2007;34:1088-96.

20. Hambye AS, Vervaet A, Dobbeleir A. Variability of left ventricular ejection fraction and volumes with quantitative gated SPECT: influence of algorithm, pixel size and reconstruction parameters in small and normal-sized hearts. Eur J Nucl Med Mol Imaging. 2004;31:1606-13.

21. Nakajima K, Taki J, Higuchi T, Kawano M, Taniguchi M, Maruhashi K, et al. Gated SPET quantification of small hearts: mathematical simulation and clinical application. Eur J Nucl Med. 2000;27:1372-9.

22. Chung AK, Das SR, Leonard D, Peshock RM, Kazi F, Abdullah $\mathrm{SM}$, et al. Women have higher left ventricular ejection fractions than men independent of differences in left ventricular volume: the Dallas Heart Study. Circulation. 2006;113:1597-604.

23. Nakajima K, Taki J, Kawano M, Higuchi T, Sato S, Nishijima C, et al. Diastolic dysfunction in patients with systemic sclerosis detected by gated myocardial perfusion SPECT: an early sign of cardiac involvement. J Nucl Med. 2001;42:183-8.

24. Kumita S, Cho K, Nakajo H, Toba M, Uwamori M, Mizumura S, et al. Assessment of left ventricular diastolic function with electrocardiography-gated myocardial perfusion SPECT: comparison with multigated equilibrium radionuclide angiography. J Nucl Cardiol. 2001;8:568-74.

25. Kikkawa M, Nakamura T, Sakamoto K, Sugihara H, Azuma A, Sawada T, et al. Assessment of left ventricular diastolic function from quantitative electrocardiographic-gated ${ }^{99 \mathrm{~m}}$ Tc-tetrofosmin myocardial SPET. Eur J Nucl Med. 2001;28:593-601.

26. Clements IP, Hodge DO, Scott CG. Frequency and determinants of early rapid filling abnormality. J Nucl Cardiol. 2006;13:53143. 
27. Taki J, Higuchi T, Nakajima K, Matsunari I, Hwang EH, Bunko $\mathrm{H}$, et al. Electrocardiographic gated ${ }^{99 \mathrm{~m}} \mathrm{Tc}-\mathrm{MIBI}$ SPECT for functional assessment of patients after coronary artery bypass surgery: comparison of wall thickening and wall motion analysis. J Nucl Med. 2002;43:589-95.

28. Giubbini R, Rossini P, Bertagna F, Bosio G, Paghera B, Pizzocaro $\mathrm{C}$, et al. Value of gated SPECT in the analysis of regional wall motion of the interventricular septum after coronary artery bypass grafting. Eur J Nucl Med Mol Imaging. 2004;31:1371-7.

29. Mabuchi M, Kubo N, Morita K, Makino Y, Matsui Y, Murashita $\mathrm{T}$, et al. Prediction of functional recovery after coronary bypass surgery using quantitative gated myocardial perfusion SPECT. Nucl Med Commun. 2003;24:625-31.

30. Akhter N, Nakajima K, Okuda K, Matsuo S, Yoneyama T, Taki J, et al. Regional wall thickening in gated myocardial perfusion SPECT in a Japanese population: effect of sex, radiotracer, rotation angles and frame rates. Eur $\mathbf{J}$ Nucl Med Mol Imaging. 2008;35:1608-15.

31. Chikamori T, Yamashina A, Hida S, Nishimura T. Diagnostic and prognostic value of BMIPP imaging. J Nucl Cardiol. 2007;14:111-25.

32. Matsuki T, Tamaki N, Nakata T, Doi A, Takahashi H, Iwata M, et al. Prognostic value of fatty acid imaging in patients with angina pectoris without prior myocardial infarction: comparison with stress thallium imaging. Eur J Nucl Med Mol Imaging. 2004;31:1585-91.

33. Tamaki N, Morita K. Is BMIPP a sensitive marker for myocardial ischaemic stress? For Eur J Nucl Med Mol Imaging. 2004;31:1031-3.

34. Matsunari I, Schricke U, Bengel FM, Haase HU, Barthel P, Schmidt $\mathrm{G}$, et al. Extent of cardiac sympathetic neuronal damage is determined by the area of ischemia in patients with acute coronary syndromes. Circulation. 2000;101:2579-85.

35. Matsuo S, Nakajima K, Yamashina S, Sakata K, Momose M, Hashimoto J, et al. Characterization of Japanese standards for myocardial sympathetic and metabolic imaging in comparison with perfusion imaging. Ann Nucl Med. 2009;23:517-22.

36. Merlet P, Pouillart F, Dubois-Rande JL, Delahaye N, Fumey R, Castaigne A, et al. Sympathetic nerve alterations assessed with ${ }^{123}$ I-MIBG in the failing human heart. J Nucl Med. 1999;40:22431.

37. Kyuma M, Nakata T, Hashimoto A, Nagao K, Sasao H, Takahashi $\mathrm{T}$, et al. Incremental prognostic implications of brain natriuretic peptide, cardiac sympathetic nerve innervation, and noncardiac disorders in patients with heart failure. J Nucl Med. 2004;45:155-63.

38. Nakata T, Wakabayashi T, Kyuma M, Takahashi T, Tsuchihashi K, Shimamoto K. Cardiac metaiodobenzylguanidine activity can predict the long-term efficacy of angiotensin-converting enzyme inhibitors and/or beta-adrenoceptor blockers in patients with heart failure. Eur J Nucl Med Mol Imaging. 2005;32:186-94.

39. Agostini D, Verberne HJ, Burchert W, Knuuti J, Povinec P, Sambuceti G, et al. I-123-mIBG myocardial imaging for assessment of risk for a major cardiac event in heart failure patients: insights from a retrospective European multicenter study. Eur J Nucl Med Mol Imaging. 2008;35:535-46.

40. Nakajima K, Yoshita M, Matsuo S, Taki J, Kinuya S. Iodine-123MIBG sympathetic imaging in Lewy-body diseases and related movement disorders. Q J Nucl Med Mol Imaging. 2008;52:378-87.

41. Estorch M, Camacho V, Paredes P, Rivera E, RodriguezRevuelto A, Flotats A, et al. Cardiac ${ }^{123}$ I-metaiodobenzylguanidine imaging allows early identification of dementia with Lewy bodies during life. Eur J Nucl Med Mol Imaging. 2008;35:1636-41.

42. Braune $\mathrm{S}$. The role of cardiac metaiodobenzylguanidine uptake in the differential diagnosis of parkinsonian syndromes. Clin Auton Res. 2001;11:351-5.
43. Taki J, Nakajima K, Hwang EH, Matsunari I, Komai K, Yoshita $\mathrm{M}$, et al. Peripheral sympathetic dysfunction in patients with Parkinson's disease without autonomic failure is heart selective and disease specific. Eur J Nucl Med. 2000;27:566-73.

44. McKeith IG. Consensus guidelines for the clinical and pathologic diagnosis of dementia with Lewy bodies (DLB): report of the Consortium on DLB International Workshop. J Alzheimers Dis. 2006;9:417-23.

45. Merlet P, Benvenuti C, Moyse D, Pouillart F, Dubois-Rande JL, Duval AM, et al. Prognostic value of MIBG imaging in idiopathic dilated cardiomyopathy. J Nucl Med. 1999;40:917-23.

46. Verberne HJ, Habraken JB, van Eck-Smit BL, Agostini D, Jacobson AF. Variations in ${ }^{123}$ I-metaiodobenzylguanidine (MIBG) late heart mediastinal ratios in chronic heart failure: a need for standardisation and validation. Eur $\mathrm{J}$ Nucl Med Mol Imaging. 2008;35:547-53

47. Yamashina S, Yamazaki J. Role of MIBG myocardial scintigraphy in the assessment of heart failure: the need to establish evidence. Eur J Nucl Med Mol Imaging. 2004;31:1353-5.

48. Nakajima K, Matsubara K, Ishikawa T, Motomura N, Maeda R, Akhter N, et al. Correction of iodine-123-labeled meta-iodobenzylguanidine uptake with multi-window methods for standardization of the heart-to-mediastinum ratio. $\mathrm{J}$ Nucl Cardiol. 2007; $14: 843-51$.

49. Nishimura T. Heart failure and cardiac sympathetic neuronal function. Tokyo: Medical View; 2002. p. 148-55. (in Japanese).

50. Verberne HJ, Feenstra C, de Jong WM, Somsen GA, van EckSmit BL, Busemann Sokole E. Influence of collimator choice and simulated clinical conditions on ${ }^{123}$ I-MIBG heart/mediastinum ratios: a phantom study. Eur $\mathrm{J}$ Nucl Med Mol Imaging. 2005;32:1100-7.

51. Chen J, Garcia EV, Galt JR, Folks RD, Carrio I. Optimized acquisition and processing protocols for I-123 cardiac SPECT imaging. J Nucl Cardiol. 2006;13:251-60.

52. Matsuo S, Nakajima K, Okuda K, Kawano M, Ishikawa T, Hosoya T, et al. Standardization of the heart-to-mediastinum ratio of ${ }^{123}$ I-labelled-metaiodobenzylguanidine uptake using the dual energy window method: feasibility of correction with different camera-collimator combinations. Eur J Nucl Med Mol Imaging. 2009;36:560-6.

53. Momose M, Tyndale-Hines L, Bengel FM, Schwaiger M. How heterogeneous is the cardiac autonomic innervation? Basic Res Cardiol. 2001;96:539-46.

54. Sakata K, Shirotani M, Yoshida H, Kurata C. Physiological fluctuation of the human left ventricle sympathetic nervous system assessed by iodine-123-MIBG. J Nucl Med. 1998;39:1667-71.

55. Nishimura T, Nakajima K, Kusuoka H, Yamashina A, Nishimura S. Prognostic study of risk stratification among Japanese patients with ischemic heart disease using gated myocardial perfusion SPECT: J-ACCESS study. Eur J Nucl Med Mol Imaging. 2008:35:319-28.

56. Nakajima K, Nishimura T. Prognostic table for predicting major cardiac events based on J-ACCESS investigation. Ann Nucl Med. 2008;22:891-7.

57. Matsuo S, Nakajima K, Horie M, Nakae I, Nishimura T. Prognostic value of normal stress myocardial perfusion imaging in Japanese population. Circ J. 2008;72:611-7.

58. Slomka PJ, Fish MB, Lorenzo S, Nishina H, Gerlach J, Berman DS, et al. Simplified normal limits and automated quantitative assessment for attenuation-corrected myocardial perfusion SPECT. J Nucl Cardiol. 2006;13:642-51.

59. Grossman GB, Garcia EV, Bateman TM, Heller GV, Johnson LL, Folks RD, et al. Quantitative Tc-99m sestamibi attenuation-corrected SPECT: development and multicenter trial validation of myocardial perfusion stress gender-independent normal database in an obese population. J Nucl Cardiol. 2004;11:263-72. 
60. O'Connor M, Kemp B, Anstett F, Christian P, Ficaro EP, Frey E, et al. A multicenter evaluation of commercial attenuation compensation techniques in cardiac SPECT using phantom models. J Nucl Cardiol. 2002;9:361-76.

61. Nishina H, Slomka PJ, Abidov A, Yoda S, Akincioglu C, Kang $\mathrm{X}$, et al. Combined supine and prone quantitative myocardial perfusion SPECT: method development and clinical validation in patients with no known coronary artery disease. J Nucl Med. 2006;47:51-8.

62. Berman DS, Kang X, Nishina H, Slomka PJ, Shaw LJ, Hayes SW, et al. Diagnostic accuracy of gated Tc-99m sestamibi stress myocardial perfusion SPECT with combined supine and prone acquisitions to detect coronary artery disease in obese and nonobese patients. J Nucl Cardiol. 2006;13:191-201.

63. Okuda K, Nakajima K, Motomura N, Kubota M, Yamaki N, Maeda $\mathrm{H}$, et al. Attenuation correction of myocardial SPECT by scatter-photopeak window method in normal subjects. Ann Nucl Med. 2009;23:501-6.

64. Slomka PJ, Cheng VY, Dey D, Woo J, Ramesh A, Van Kriekinge $\mathrm{S}$, et al. Quantitative analysis of myocardial perfusion SPECT anatomically guided by coregistered 64-slice coronary CT angiography. J Nucl Med. 2009;50:1621-30.

65. Gaemperli O, Schepis T, Kalff V, Namdar M, Valenta I, Stefani $\mathrm{L}$, et al. Validation of a new cardiac image fusion software for three-dimensional integration of myocardial perfusion SPECT and stand-alone 64-slice CT angiography. Eur J Nucl Med Mol Imaging. 2007;34:1097-106.

66. Matsuo S, Nakajima K, Akhter N, Wakabayashi H, Taki J, Okuda $\mathrm{K}$, et al. Clinical usefulness of novel cardiac MDCT/SPECT fusion image. Ann Nucl Med. 2009;23:579-86 